\title{
The Francis Crick Institute
}

\author{
Authors: Keith Peters ${ }^{A}$ and Jim Smith ${ }^{B}$
}

The Francis Crick Institute Laboratory, opened in 2016, is supported by the Medical Research Council, Cancer Research UK, the Wellcome Trust, and University College London, King's College London and Imperial College London. The emphasis on research training and early independence of gifted scientists in a multidisciplinary environment provides unique opportunities for UK medical science, including clinical and translational research.

KEYWORDS: MRC, CRUK, Wellcome, Crick, UCL, King's, Imperial

The Francis Crick Institute has been referred to as the most significant development in UK biomedical science for a generation. It has been built at a cost of nearly $£ 700$ million and is directed by Nobel Laureate and geneticist Sir Paul Nurse. What will it contribute to biomedicine, and how will it do it?

\section{History}

The Crick arose from discussions in the Medical Research Council (MRC) about the future of the National Institute for Medical Research (NIMR), the MRC's largest research institute. The NIMR's laboratory in Mill Hill was getting old and its location - far from a university, medical school or teaching hospital - made it difficult to fulfil the MRC's scientific and translational aspirations. A particular concern was the need to provide a laboratory base for medical scientists with clinical duties and also to have a laboratory of sufficient scale to allow joint research with industry scientists. The search for a new site ended with the identification of land to the north of the British Library and to the west of St Pancras station. This was agreed to be an excellent location, close to many London universities and to first-rate general and specialist hospitals, such as Great Ormond Street Hospital, Moorfields Eye Hospital and the National Hospital for Neurology and Neurosurgery, Queen Square. Recently, the Alan Turing Institute has started work in the British Library and is due to be accommodated in a purpose-designed building next to the Crick. Transport links are superb - with trains, tubes and buses close by - and, notably, the institute

Authors: A consultant to the Francis Crick Institute, London, UK; ${ }^{B}$ former director, Francis Crick Institute, London, UK and head of science, the Wellcome Trust, London, UK is within easy reach of GlaxoSmithKine (GSK) and Astra Zeneca's principal research laboratories in Stevenage and Cambridge, respectively.

But in order to justify investment on the scale required, the new institute needed to be more than a simple translocation to a new site.

After discussion and negotiation, the MRC, Cancer Research UK (CRUK), the Wellcome Trust and University College London (UCL) created a partnership: CRUK's London Research Institute (LRI) would join the NIMR, with researchers from UCL contributing expertise in the physical and clinical sciences. Importantly, the Crick was not simply to be a merger of LRI and NIMR but a new entity with a different ethos - a multidisciplinary institute with a substantial new investment in the biomedically related physical sciences, which, through the clinical sciences, would place increased emphasis on research in human biology and its perturbations in disease.

Such was the excitement surrounding the initiative that King's College and Imperial College London joined as full partners in 2011; the Crick now had six partners all contributing to the cost of the new building. The MRC and CRUK transferred the running costs of their former institutes to the Crick, and the Wellcome Trust made a strategic award of $\mathfrak{E} 70$ million over 5 years in addition to a major contribution to the capital development.

The Francis Crick Institute became a legal entity on 1 April 2015, when researchers from the NIMR and LRI transferred to the employment of the Crick. The next major event was the completion and handover of the Crick's building in August 2016, followed by its opening by Her Majesty the Queen on 9 November.

\section{The building}

It is impossible to write about the Crick without mentioning its magnificent building (Fig 1). The remit of the architects was challenging. The new building had to accommodate almost 1,300 researchers and about 200 administrative and support staff; be capable of housing the most sensitive scientific equipment; provide a substantial animal facility; and include containment facilities that would allow research to be carried out on the most virulent strains of influenza. The central London site had to relate to the British Library to the south and to St Pancras station to the east, and be sensitive to the concerns of those living in the social housing to the north and west. Although the plot was larger than that required by NIMR, the introduction of other partners made space tight. 


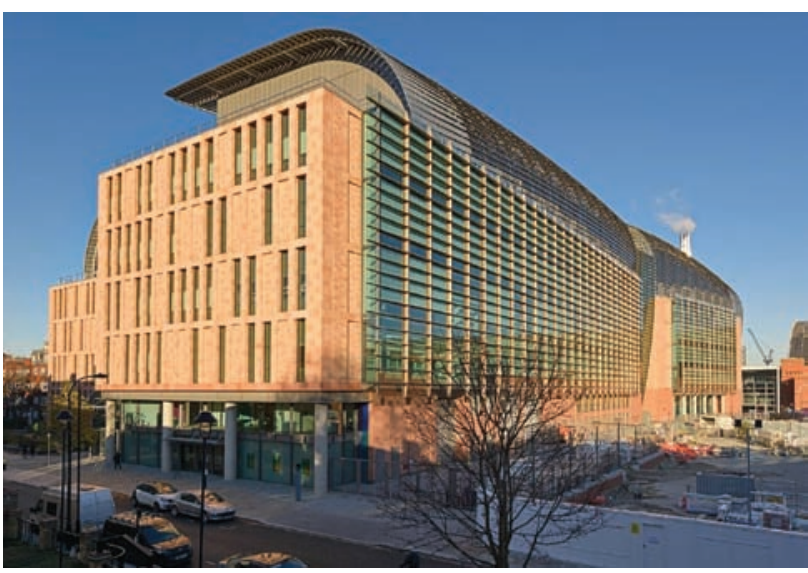

Fig 1. The Francis Crick Institute. @ Francis Ware 2016

One solution adopted by the architects (HOK and PLP Architecture) was to dig down, so one third of the building is positioned underground. The apparent mass above ground was reduced by placing the curved roof (which echoes the Barlow Shed of St Pancras) away from the main walls, such that it is not visible from the street. Most importantly, the building is open - open to the public and open to the researchers inside. The public have access to the exhibition space and they can see scientists working in the building: having coffee or lunch, discussing their results and reading papers. Scientists work in large open-plan lab spaces. There are clear lines of sight so that it is easy to see colleagues; the architects were made aware of the desirability of promoting chance interactions and a prominent feature is the large central staircase that people will use several times a day. Of particular importance, there are large collaboration spaces in the centre of each floor, and on the bridges that connect the labs across the atrium. In some respects the building is an experiment in social engineering and the impression so far is that the experiment is working.

\section{What makes the Crick different?}

The Crick inherits great scientific momentum from the NIMR and LRI but as its new identity evolves, it will differ from similarly sized institutions. First, its scale means that it will not need to focus on any one subject, such as tumour immunology, infectious disease or regenerative medicine. Consistent with this approach, the institute will have no departments or divisions to drive strategy, and the lack of departments will also encourage research groups to interact with colleagues in different research areas. As described above, the Crick's building is designed to promote interactions. The focus of the institute will be research excellence with an emphasis on securing outstanding early-career researchers with little heed to their subject area. The Crick intends to use its independence to ignore the usual journal-based metrics of success and appoint and assess on the basis of the importance of the questions they are addressing and their potential to answer them.

The Francis Crick Institute's career structure will also reflect and encourage this strategy. Early-career researchers will be recruited, in the first instance, for 6 years. They will receive generous core support with no need, in their first few years, to apply for external grants. After 6 years, subject to review, they will be eligible to remain for 6 more years, after which they will leave the institute for senior positions elsewhere. No exceptions will be made, which will ensure that the institute has turnover, maintains its excellence and fulfils its national role by attracting the best scientists to the UK, by providing the best training possible and then placing them in the best institutions in the country. One of the ways the Crick will measure success is not by asking who it retains, but who leaves and where they go. This career structure is designed to maintain excellence, allow the Crick to be nimble in its science and enable the institute to fulfil its national role as research and health priorities and opportunities evolve.

As a new and independent institute, the Crick has an opportunity to set bold precedents in its recruitment strategy, and one area in which the institute hopes to excel is equality and diversity. The Crick has no intention to lose the best scientists because they are women, or gay, or come from the 'wrong' ethnic or social background. Unconscious bias training will be undertaken and part-time working will be supported.

International recruitment will be key to the Crick's success and, indeed, to the success of UK biomedicine. However, a threat to both is Brexit.

\section{The Greater Crick}

The Crick differs from other institutes in its constitution: it has three parent universities and their associated medical schools and health providers. It is this link with the London universities that will be key to the expansion of its scientific horizons - in the clinical sciences and in subjects such as chemistry, material science and the data sciences. Researchers from the universities will be encouraged to take up short- to medium-term appointments in the Crick. These may be through sabbaticals, satellite groups (where a university-based group leader sends group members to the institute to work with a Crick group leader) or secondments (where an entire university-based group moves to the Crick for a period of up to 6 years). The Crick partners have been through two rounds of this process so far, which has already succeeded in bringing new types of science to the institute. A reciprocal arrangement is planned for the Crick to enable its researchers to work in partner university departments, a process that should begin next year.

The Crick and its partners are enthusiastic about these interactions, which inspired joint activities even before the building was completed. These have included the Crick PhD programme, in which all students have a university-based co-supervisor. Shared supervision has proved to be a powerful way to encourage interactions. There has been an inspiring series of interdisciplinary symposia on wide-ranging subjects, such as imaging, tuberculosis and mathematical biology; these meetings will soon be complemented by joint symposia with the Alan Turing Institute.

There has been little opportunity, at this early stage of the Institute's history, to expand internationally beyond links already established in the parent research institutes. However, the Crick's proximity to the London School of Hygiene and 
Tropical Medicine should catalyse new programmes in global health. The potential of developments along these lines has given rise to the concept of the Greater Crick - the Greater Crick embraces the partnerships and promotes interactions between them; and, importantly, envisages the Francis Crick Institute as a functional entity beyond the physical confines of the new laboratory, harnessing the full potential of London's biomedical science.

\section{Clinical science}

The Francis Crick Institute is dedicated to understanding the scientific mechanisms of living things, with the objectives of understanding why disease develops and of finding new ways to treat, diagnose and prevent illnesses such as cancer, heart disease, stroke, infections and neurodegenerative diseases. To achieve these aims, the Crick is adopting multidisciplinary approaches and, as already emphasised, plans to engage in clinical research in partnership with the London medical schools. In particular, it hopes to shift its research emphasis towards human biology, the essential basis of translational medicine.

To this end, the Crick appointed one of us (KP) to advise the Crick on clinical and translational work in the years leading to the official opening and he has now been succeeded as clinical research director by Sir Peter Ratcliffe from Oxford. Ratcliffe's research, for which he has lately been presented with the 2016 Lasker Award, concerns the mechanisms by which cells sense oxygen levels to direct a range of adaptive responses that are important in cancer, cardiovascular and metabolic disease. As a clinician, he joins Akilesh Reddy, a neurologist recruited from the University of Cambridge to continue his studies of circadian rhythms, Rob Wilkinson, who studies patients in South Africa who are infected with tuberculosis and HIV, Charlie Swanton, a cancer researcher exploring genetic diversity in tumours and its consequences on clinical outcome, and Peter Oppenshaw from Imperial, who has a long standing collaboration with the NIMR in infectious disease. These senior clinicians will work with researchers from the three universities and recruit clinical research fellows. Medical students from UCL's MB/PhD programme will be encouraged to undertake their research in the institute's laboratories. It is intended to institute a regular Crick Grand Round, in partnership with the three medical schools.

Other initiatives are being explored in which the Crick collaborates with medical schools around the UK. For example, applications for renewals of the English Biomedical Research Centres have the opportunity to propose a 'buddy' system, in which Crick non-clinical group leaders partner with clinician researchers. And the N4 group of universities - Leeds, Manchester, Newcastle and Sheffield - have set up a programme with support from the Wellcome Trust that enables their best clinical PhD students to spend time in the Crick to develop their science and be in a strong position to compete for career development fellowships in their parent medical schools. It is anticipated that these various initiatives will create a more medical mindset in the institute.

\section{Translation}

A key objective for the Francis Crick Institute is to facilitate the translation of discovery science. This is a challenging and difficult process and the research emphasis at the Crick is on basic biomedical science. However, in order to ensure that translational opportunities are not lost, the Crick has introduced an innovative programme in which a team led by David Roblin, a medically qualified and distinguished pharmaceutical scientist, ensures that the potential of the Crick discovery research is recognised and that arrangements for its translation, often in association with industry partners, are smoothly and efficiently expedited. The mindset that is being promoted is that the Crick is open to translation. In the same way that a critical mass of clinicians can 'medicalise' a basic research institute, a critical mass of industry researchers is needed to provide expertise in translational science and enhance awareness of translational opportunities. To this end, the Crick has created an open innovation partnership with GSK, the UK's largest pharmaceutical company. The partnership began with an 'awayday' enabling informal interactions between industry and institute scientists and has now evolved into a 'Linklab' partnership in which GSK scientists work in the Crick alongside Crick researchers, and Crick scientists work in GSK's hub in Stevenage. The Crick and GSK both contribute resources to the partnership, including space, personnel, equipment, reagents and consumables.

The partnership benefits from the proximity of GSK's research labs at Stevenage and the institutions' complementary areas of expertise. Importantly, intellectual property belongs to the Crick and research findings from the collaboration are to be published in peer-reviewed journals so that the discoveries can be accessed by the research community. The shared objective is accelerating the development of new treatments.

The partnership is already a success. From a standing start, there are now nine collaborative projects in the areas of infectious disease, including malaria and tuberculosis, and four in oncology. It is anticipated that the success of this scheme will soon attract similar arrangements with the research laboratories of other healthcare companies.

\section{Conclusions}

The Crick is a magnificent resource for UK medicine. Its research builds on the distinguished record of its founding institutes but its strategy and constitution - particularly the Greater Crick - have unique potential to develop the careers of the most talented researchers and to address some of the most important and challenging questions in biomedical science.

\section{Conflicts of interest}

KP was a senior consultant to GlaxoSmithKline from 2005-2016. JS is the science director for the Wellcome Trust.

Address for correspondence: Sir Keith Peters, Francis Crick Institute, 1 Midland Road, London NW1 1AT, UK.

Email: keith.2.peters@crick.ac.uk 